\title{
¿Crear y compartir conocimiento motiva a nuestro alumnado? Does creating and sharing knowledge motivate our students?
}

\author{
Ángel Fidalgo Blanco ${ }^{1}$, María Luisa Sein-Echaluce Lacleta ${ }^{2}$, Laura García Ruesgas ${ }^{3}$, David Fonseca ${ }^{4}$ \\ angel.fidalgo@upm.es,_mlsein@unizar.es, lauragr@us.es, david.fonseca@salle.url.es \\ ${ }^{1}$ Departamento de Ingeniería Geológica y Minera \\ Universidad Politécnica de Madrid \\ Madrid, España \\ ${ }^{3}$ Departamento de Ingeniería Gráfica \\ Universidad de Sevilla \\ Sevilla, España \\ ${ }^{2}$ Departamento de Matemática Aplicada \\ Universidad de Zaragoza \\ Zaragoza, España \\ ${ }^{4}$ Departamento de Arquitectura \\ Universidad Ramón Llull, La Salle \\ Barcelona, España
}

\begin{abstract}
Resumen- La creación de conocimiento por parte del alumnado se asocia a la utilización de capacidades cognitivas altas que favorecen el proceso de aprendizaje. Desde el punto de vista de las organizaciones, la creación de conocimiento por parte de sus miembros se asocia a la creación de conocimiento organizacional, que hace a la organización más competitiva. En este trabajo el alumnado crea conocimiento relacionado con la asignatura para mejorar su aprendizaje individual e incrementar el conocimiento de la propia asignatura. Para tener un primer análisis de los puntos fuertes y débiles de este modelo docente aplicado, se ha utilizado la encuesta MUSIC, que mide varias dimensiones relacionadas con la motivación, con alumnado que ha experimentado con el modelo descrito. Los resultados obtenidos arrojan que los puntos fuertes están mas relacionados con las personas y los puntos débiles con el interés que suscita este modelo por parte del alumnado.
\end{abstract}

Palabras clave: motivación de los estudiantes, innovación educativa, sistema de gestión de conocimiento

Abstract- The creation of knowledge by students is associated with the use of high cognitive capacities that favor the learning process. From the point of view of organizations, the creation of knowledge by their members is associated with the creation of organizational knowledge, which makes the organization more competitive. In this research students create knowledge that is related to the subject in order to improve their individual learning and increase the knowledge of the subject itself. In order to have a first analysis of the strengths and weaknesses of this applied teaching model, the MUSIC survey has been used, which measures several dimensions related to motivation, with students who have experimented with the described model. Obtained results show that the strong points are more related to people and the weak ones to the interest that this model causes among students. Keywords: students' motivation, educational innovation, knowledge
managemen system

\section{INTRODUCCIÓN}

Desde el punto de vista del aprendizaje, este se asocia a la adquisición de distintos niveles cognitivos, siendo éstos de distinto orden. Bloom desarrolló una taxonomía para la evaluación del nivel cognitivo adquirido en el proceso de aprendizaje (Bloom, Engelhart, Furst, Hill, \& Krathwohl,

1956). La taxonomía consta de niveles de orden inferior (aprendizaje con bajo nivel cognitivo como conocimiento, comprensión y aplicación) y de orden superior (aprendizaje con alto nivel cognitivo como análisis, síntesis y evaluación).

En revisiones posteriores de dicha taxonomía se agrupan los niveles para los procesos cognitivos y para el conocimiento. La pirámide en este caso tiene en su cúspide la creación de conocimiento (Krathwohl, 2002).

Así mismo, se modificó la taxonomía de Bloom para adaptarla a la era digital (Churches, 2009) y, en esa adaptación se asocian las actividades cognitivas con procesos y tecnologías que son propias de la sociedad del conocimiento, como: buscar información, subir archivos a la nube, comentar un blog, participar en una red social y que el propio alumnado cree y publique conocimiento.

Por otra parte, atendiendo a las modalidades de aprendizaje oficiales en los distintos entornos académicos, durante el curso académico 2020-2021, y debido a la pandemia COVID-19, todavía la mayor parte de las asignaturas se impartieron en modalidad totalmente virtual. Esta situación da lugar a la aplicación de la taxonomía de Bloom, modificada para la era digital, a los entornos educativos donde la creación de contenidos y su publicación llevan consigo los mayores niveles cognitivos por parte de las personas que los llevan a cabo.

Así pues, en el mundo educativo se asocia el aprendizaje al nivel cognitivo de creación de conocimiento; es decir, la creación de conocimiento produce un nivel cognitivo de aprendizaje alto. Sin embargo, desde el punto de vista del aprendizaje organizacional, este planteamiento es el inverso. El aprendizaje, entre otros factores, potencia la creación de conocimiento y este se convierte en capital que genera valor para la organización (Barragán Ocaña, 2009).

Los principios de aprendizaje organizacional se ligan a una organización que aprende (Nonaka \& Takeuchi, 1995). En este trabajo el planteamiento es considerar a una asignatura académica como una organización que aprende y en la que, además, sus miembros también aprenden generando valor para la propia asignatura. 
La creación de conocimiento por parte del alumnado tiene dos puntos de vista: el educativo donde el valor se pone en el aprendizaje del individuo y el organizacional donde el valor se pone en el aprendizaje de la organización. El planteamiento de este trabajo se basa en unir el punto de vista educativo y el organizacional en lo que respecta a la creación de conocimiento. De esta forma, a través de esta acción el propio alumnado adquiere un nivel cognitivo alto de aprendizaje y la asignatura aumenta el conocimiento ya que incorpora la experiencia del alumnado.

La motivación está muy relacionada con el compromiso del estudiante, con los resultados de aprendizaje (Howard, Bureau, Guay, Chong, \& Ryan, 2021) y las aptitudes del alumnado (González Pérez \& Fabiani Bendicho, 2019) y sirve como medida de modelos formativos basados en innovación docente (Efrén Mora, Añorbe Díaz, González Marrero, Martín Gutiérrez, \& Jones, 2017).

Una herramienta validada para medir la motivación del estudiante es la encuesta "eMpowerment, Usefulness, Success, Interest, and Caring” (MUSIC) (Jones, 2009). En este trabajo se analiza la motivación del estudiante, medida por la encuesta MUSIC, y los resultados de la encuesta se utilizan para analizar los puntos débiles y fuertes de la unión del punto de vista educativo y organizacional, a través de la creación de conocimiento por parte del alumnado.

El objetivo de esta investigación es, por tanto, utilizar la motivación como un primer indicador de las fortalezas y debilidades del modelo de creación de conocimiento propuesto, tanto a nivel del aprendizaje individual (el estudiante), como del organizacional (la asignatura).

En la siguiente sección se describe el modelo funcional que combina el aprendizaje individual a través de la creación de contenidos con el organizacional. Posteriormente se describe el contexto de aplicación de la investigación para continuar con el análisis de resultados y conclusiones.

\section{MODELO}

En esta sección se incluye, en primer lugar, la descripción de un modelo docente (RT-CICLO + ACCI 3.0) y en la segunda parte se realizará la inclusión de este modelo con la encuesta MUSIC.

\section{A. Modelo docente propuesto}

El modelo utilizado es fruto de la unión de un modelo procedimental para la creación de conocimiento compartido y que se denomina RT-CICLO (Real Time - Collective Intelligence applied to a Cooperative Learning with a sOcial base)(Ángel Fidalgo-Blanco, Sein-Echaluce, \& GarcíaPeñalvo, 2018; García-Peñalvo, Fidalgo-Blanco, SeinEchaluce, \& Sánchez-Canales, 2019), con un framework tecnológico denominado ACCI 3.0 (Active Cooperative Collective Intelligence bajo un modelo Web 3.0) (Á. FidalgoBlanco, García-Ruesgas, Fernández Blanco, \& Sastre-Merino, 2020).

Ambos modelos, de forma integrada, constituyen un método de aprendizaje organizacional que ya se ha aplicado a una asignatura (Á. Fidalgo-Blanco, Sein-Echaluce, \& García-
Peñalvo, 2017; Ángel Fidalgo-Blanco, Sánchez-Canales, SeinEchaluce, \& García-Peñalvo, 2018). La Figura 1 representa la integración de los modelos RT-CICLO y ACCI 3.0.

El modelo RT-CICLO (partes rojas de la Figura 1) representa los distintos procesos relacionados con la creación de conocimiento por parte del alumnado. Durante las actividades de aprendizaje de la asignatura (clases teóricas, prácticas, laboratorios y cualquier otra actividad de aprendizaje) el alumnado adquiere conocimiento a distintos niveles. Pueden tener un aprendizaje profundo de conocimiento o aprendizajes superficiales y, en otras ocasiones, no se habrá producido aprendizaje de ningún tipo, bien porque no han entendido los conceptos, porque estaban distraídos, por no tener suficientes conocimientos previos, etc.

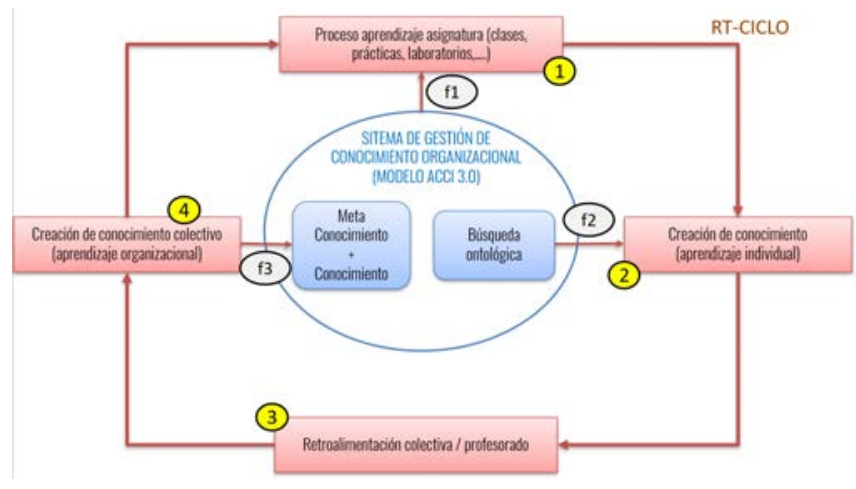

Figura 1. Modelos RT-CICLO y ACCI 3.0

En el modelo docente propuesto el alumnado forma equipos de trabajo y, una vez formados, comienzan las fases del modelo RT-CICLO.

El alumnado adquiere aprendizaje a través de las distintas actividades docentes realizadas en la asignatura (clases teóricas, prácticas, laboratorios, etc.) La fase 1 del modelo está relacionada con las actividades de aprendizaje diarias. De forma periódica el equipo se reúne y analiza el aprendizaje de los componentes del equipo. A partir de este análisis se identifica el conocimiento a crear. Por ejemplo, puede ser un concepto que casi nadie del equipo ha entendido, o un ejercicio que consideran importante para el desarrollo de la asignatura (Figura 1-1).

Durante la segunda fase (Figura 1-2) el alumnado elabora recursos de aprendizaje. Es en esta fase donde comienza a producirse el aprendizaje individual, ya que habitualmente el alumnado que no ha entendido un concepto determinado, o cuando ha tenido un aprendizaje superficial, elabora materiales relacionados con esos conceptos De esta forma se produce un aprendizaje a nivel individual con un alto nivel cognitivo (Bloom et al., 1956; Churches, 2009; Krathwohl, 2002). El conocimiento creado en esta fase aún no está validado; es decir, una vez creado hay que comprobar que cumple con la normativa de propiedad intelectual, que son correctos los datos y que no hay ningún error conceptual ni de desarrollo. Todo ello se realiza en la siguiente fase.

En la tercera fase (Figura 1-3) se comprueba la calidad del recurso elaborado. Para ello, es el equipo quien revisa el conocimiento creado. Se establecen dos niveles de 
comprobación (elegibles por el equipo). En un nivel todos los miembros del equipo comprueban el conocimiento que se ha creado (la comprobación por parte de los miembros que no han creado el conocimiento, tiene especial valor). Otro nivel es la comprobación por parte del profesorado, labor que se suele realizar durante las sesiones de tutoría. En la siguiente fase tiene lugar la elaboración del conocimiento final.

Para finalizar el proceso de creación de conocimiento, el equipo añade meta información (Figura 1-4) a lo creado en la fase anterior. Esta meta información consta de: el tipo de recurso (ejemplo, apuntes, problema resuelto, etc.), un resumen explicativo, recomendaciones de uso del recurso y la justificación por la que han elaborado dicho recurso. Toda esta meta información constituye un aspecto clave para la creación de conocimiento organizacional, ya que facilita la organización, clasificación y búsqueda del conocimiento creado.

El modelo ACCI 3.0 (partes azuladas de la Figura 1) es la parte de software que se encarga de gestionar el conocimiento: almacenamiento, clasificación, organización y búsqueda. Se compone de dos programas software: uno es Wordpress (sistema de gestión documental) y otro es un buscador por ontologías, sistema de desarrollo propio que se encarga de la clasificación y las búsquedas.(Á. Fidalgo-Blanco et al., 2020). El alumnado utiliza el sistema ACCI 3.0 tanto para acceder a recursos ya existentes como para incluir nuevos. El conocimiento se forma a través de los recursos elaborados por el alumnado, junto con diversa meta-información como: resumen del contenido, recomendaciones de uso, verificación de la calidad y etiquetas que definen su clasificación.

Los flujos f1 y f2 de la figura 1 representan la utilización del conocimiento por parte del alumnado. El flujo f1 muestra cómo el sistema de gestión de conocimiento sirve para complementar las actividades de aprendizaje realizadas en la asignatura. En el flujo $\mathrm{f} 2$ el sistema de gestión de conocimiento ofrece recursos que ayudan al alumnado en la elaboración de nuevo conocimiento. El flujo $\mathrm{f} 3$ de la figura 1 representa la carga del conocimiento definitivo que ha creado el alumnado durante la fase 4 del modelo RT-CICLO. La carga de conocimiento se introduce a través del software de Wordpress. La elaboración del conocimiento por parte del alumnado y su utilización produce un aprendizaje entre iguales (Ángel Fidalgo-Blanco, Sein-Echaluce, \& García-Peñalvo, 2017; García-Peñalvo et al., 2019; Sein-Echaluce, Fidalgo-Blanco, Esteban-Escaño, \& García-Peñalvo, 2017)

\section{B. Integración del modelo docente propuesto con MUSIC}

La inclusión en este modelo funcional (RT-CICLO + ACCI 3.0) con el cuestionario MUSIC se muestra en la Figura 2. A la Figura 1, que muestra el modelo docente propuesto, se añaden (en verde) cada una de las letras que componen la palabra MUSIC, y que expresan las dimensiones que aborda dicha encuesta de motivación. La figura 2 muestra la relación entre esas dimensiones y las distintas etapas /actividades que se realizan en el modelo docente propuesto en el anterior subapartado.

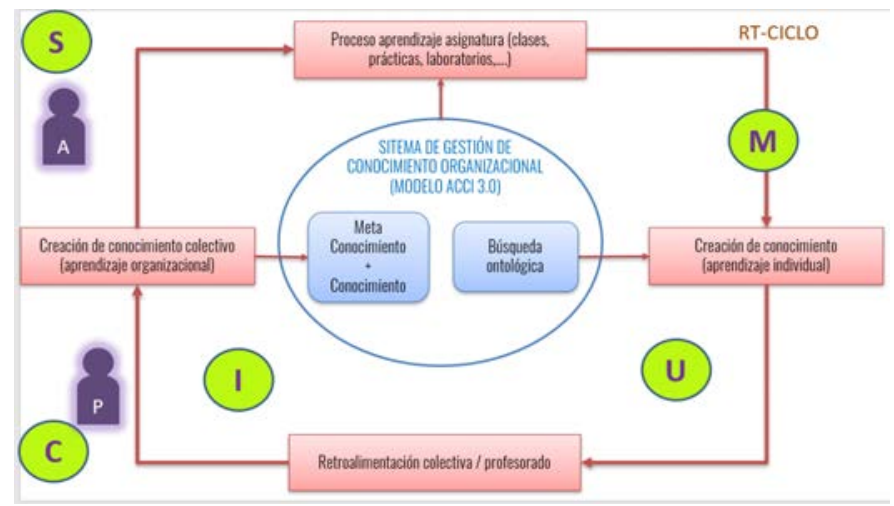

Figura 2. Intregración modelo docente con MUSIC

La herramienta de medición de los resultados de motivación es MUSIC, a través de "User guide for assessing the components of de MUSIC model of academic motivation" (Jones, 2014) esta herramienta contiene 5 dimensiones:

- $\quad M$ ("eMpowerment”). Sentirse empoderado para que el alumnado tome decisiones sobre su propio aprendizaje.

- En nuestro modelo la dimensión M se asocia a la capacidad de decisión que tiene el alumnado para elegir el conocimiento que desea elaborar y profundizar en su aprendizaje.

- $\quad U$ (Usefulnes). Percepción del estudiante sobre la utilidad de las actividades y contenidos que realizan en el curso.

- En este caso la dimensión U se asocia a la utilidad de las actividades a realizar y el conocimiento que genera el alumnado.

- $\quad S$ (Success). Percepción del éxito que van a tener en función de la dedicación y esfuerzo que están realizando en la asignatura.

- La dimensión $\mathrm{S}$ se asocia a la visión que tiene el alumnado sobre sí mismo, concretamente en la posibilidad de conseguir aprobar y aprender dentro de la asignatura.

- $I$ (Interest). Interés que suscitan los contenidos y actividades del curso.

- Al igual que en la utilidad, el interés (dimensión I) se asocia al conjunto de actividades y contenidos del curso.

- $\quad C$ (Caring). Atención que recibe el alumnado en su proceso de aprendizaje, principalmente la atención recibida por el profesorado.

- Esta dimensión $\mathrm{C}$ se asocia al trabajo que ha realizado el profesorado tanto para validar el conocimiento creado por el alumnado como para apoyarle durante las labores de tutoría e impartición de clases.

\section{CONTEXTO}

Este trabajo se ha realizado durante el curso 20-21 en la asignatura de Fundamentos de Programación correspondiente al 
primer curso del Grado de Biotecnología de la Universidad Politécnica de Madrid. La asignatura constaba de dos grupos de docencia, con un total de 96 estudiantes, que participaron en la experiencia. La modalidad de impartición de la asignatura fue totalmente virtual, debido a las restricciones impuestas por la pandemia Covid-19.

\section{Resultados}

La encuesta MUSIC consta de 26 preguntas (Tabla 1) que se contestan con la elección de 6 opciones en escala Likert de 1 a 6 (1- Nada de acuerdo a 6- Muy de acuerdo). La agrupación de las preguntas por dimensiones (aspecto a medir) se indican en la Tabla 2

Tabla 1. Encuesta MUSIC. Fuente: Jones D. User Guide for assessing the components of the MUSIC model of academic motivation

\section{Preguntas ordenadas}

1. The coursework held my attention.

2. I had the opportunity to decide for myself how to meet the course goals.

3. In general, the coursework was useful to me.

4. The instructor was available to answer my questions about the coursework.

5. The coursework was beneficial to me.

6. The instructional methods used in this course held my attention.

7. I was confident that I could succeed in the coursework.

8. I had the freedom to complete the coursework my own way.

9. I enjoyed the instructional methods used in this course.

10. I felt that I could be successful in meeting the academic challenges in this course.

11. The instructional methods engaged me in the course.

12. I had options in how to achieve the goals of the course.

13. I enjoyed completing the coursework.

14. I was capable of getting a high grade in this course.

15. The coursework was interesting to me.

16. The instructor was willing to assist me if I needed help in the course.

17. I had control over how I learned the course content.

18. Throughout the course, I felt that I could be successful on the coursework.

19. I found the coursework to be relevant to my future.

20. The instructor cared about how well I did in this course.

21. I will be able to use the knowledge I gained in this course.

22. The instructor was respectful of me.

23. The knowledge I gained in this course is important for my future.

24. The instructor was friendly.

25. I believe that the instructor cared about my feelings.
26. I had flexibility in what I was allowed to do in this course.

Tabla 2. Agrupación preguntas de encuesta MUSIC por dimensión a medir. Fuente Jones D. User Guide for assessing the components of the MUSIC model of academic motivation

\begin{tabular}{cc}
\hline Dimensión a medir & Pregunta \\
\hline M (empoderamiento) & $2,8,12,17,26$ \\
U (utilidad) & $3,5,19,21,23$ \\
S (éxito) & $7,10,14,18$ \\
I (interés) & $1,6,9,11,13,15$ \\
C (atención) & $4,16,20,22,24,25$ \\
\hline
\end{tabular}

La encuesta fue cumplimentada por 83 estudiantes de un total de 96; es decir, el $86,45 \%$ de os estudiantes del curso. Los resultados obtenidos, agrupados por dimensiones, se muestran en la Tabla 3

Tabla 1. Resultados para cada dimensión

\begin{tabular}{cc}
\hline Dimensión & Media (sobre 6) \\
\hline M & 4,45 \\
U & 4,29 \\
S & 4,58 \\
I & 3,85 \\
C & 5,27 \\
\hline
\end{tabular}

\section{CONCLUSIONES}

En este trabajo se han relacionado las distintas etapas de un modelo docente propuesto, basado en la creación de conocimiento, con las dimensiones de la encuesta de motivación MUSIC, que se ha cumplimentado para una experiencia real.

Las dimensiones de MUSIC se pueden agrupar atendiendo a su relación con las personas $(\mathrm{M}$ y $\mathrm{S}$ se refieren al alumnado y $\mathrm{C}$ al profesorado) y a su relación con el modelo (U e I se refieren a las actividades realizadas y contenidos).

En la experiencia incluida en este trabajo, las dimensiones relacionadas con el alumnado alcanzan un valor medio similar, $\mathrm{M}$ con 4,45 y S con 4,58. Esto indica que el modelo propuesto para el fomento del aprendizaje individual y organizacional, basado en la creación del conocimiento por parte del alumnado, tiene una valoración alta, tanto en la libertad para la toma de decisiones sobre su propio aprendizaje, como sobre la confianza que les aporta para obtener éxito en la asignatura. También, dentro de este mismo grupo, se ha valorado de forma muy alta $(5,27)$ el acompañamiento que ha realizado el profesorado al alumnado (dimensión $\mathrm{C}$ ).

Así pues, basándonos en el modelo MUSIC, se puede afirmar que la parte que más valora el alumnado del método propuesto es su implicación con las personas y la interacción alumnadoprofesorado. 
Respecto al otro agrupamiento, el que se refiere más a la metodología y al desarrollo de contenidos se obtienen resultados dispares (dimensiones U e I). De la tabla 3 se deduce que el alumnado considera útil las actividades y contenidos de la asignatura (dimensión $U$ ), pero no despierta su interés (dimensión I) en la misma medida que la utilidad. Esto puede ser debido a que la utilidad se relaciona con la obtención del éxito de la asignatura, mientras que el interés se asocia más a las emociones del propio alumnado.

La utilización de MUSIC para validar el modelo arroja que los puntos fuertes del modelo docente propuesto se encuentran en la interacción entre el profesorado y alumnado, así como la consideración de que lo hecho repercute en la obtención de éxito, por ejemplo, aprobando la asignatura.

El punto débil del modelo radica en la necesidad de captar el interés del propio alumnado principalmente por el modelo instruccional y, por tanto, hay que mejorarlo

Con todo lo anterior, se ha demostrado la validez del cuestionario MUSIC como elemento de obtención de puntos fuertes y débiles de un método de innovación educativa.

Como trabajo futuro se trabajará en la mejora del interés del alumnado en el modelo propuesto en este trabajo, añadiendo herramientas cualitativas como focus group y se continuará utilizando MUSIC como valoración de los puntos fuertes y débiles de otras innovaciones educativas.

\section{AgRADECIMIENTOS}

Este trabajo ha sido parcialmente apoyado por el Ministerio español de Ciencia e Innovación a través del proyecto AVisSA [PID2020-118345RB-I00] y el proyecto MECOVA [IE1920.0601] de la Universidad Politécnica de Madrid. Los autores quieren agradecer el apoyo de los grupos de investigación LITI (http://www.liti.es) y EtnoEdu (https://socioconstructivismo.unizar.es).

\section{REFERENCIAS}

Barragán Ocaña, A. (2009). Aproximación a una taxonomía de modelos de gestión del conocimiento. Intangible Capital, ISSN-e 1697-9818, Vol. 5, No. 1, 2009, Págs. 65-101, 5(1), 65-101. Retrieved from https://dialnet.unirioja.es/servlet/articulo?codigo=29097 54

Bloom, B. S., Engelhart, M. D., Furst, E. J., Hill, W. k., \& Krathwohl, D. (1956). Taxonomy of educational objectives: The classification of educational goals. Handbook I: Cognitive domain. In Taxonomy of educational objectives: The classification of educational goals. Handbook I: (pp. 201-207). New York, New York, USA: David McKay Company.

Churches, A. (2009). Taxonomía de Bloom para la Era Digital. Retrieved September 1, 2021, from Eduteka website: http://eduteka.icesi.edu.co/articulos/TaxonomiaBloomDi gital

Efrén Mora, C., Añorbe Díaz, B., González Marrero, A. M., Martín Gutiérrez, J., \& Jones, B. D. (2017). Motivational factors to consider when introducing problem-based learning in engineering education courses. The International Journal of Engineering Education, 33(3), 1000-1017. Retrieved from https://portalciencia.ull.es/documentos/5ea21c2f299952 $1 \mathrm{f} 7 \mathrm{~d} 5241 \mathrm{cf}$

Fidalgo-Blanco, Á., García-Ruesgas, L., Fernández Blanco, P., \& Sastre-Merino, S. (2020). ACCI 3.0. Technique of classification, organization, creation and use of collective knowledge. ACM International Conference Proceeding Series. https://doi.org/10.1145/3434780.3436594

Fidalgo-Blanco, Á., Sein-Echaluce, M. L., \& García-Peñalvo, F. J. (2017). Ontological Flip Teaching: a Flip Teaching model based on knowledge management. Universal Access in the Information Society. https://doi.org/10.1007/s10209-017-0556-6

Fidalgo-Blanco, Ángel, Sánchez-Canales, M., Sein-Echaluce, M. L., \& García-Peñalvo, F. J. (2018). Ontological Search for Academic Resources. Proceedings of the Sixth International Conference on Technological Ecosystems for Enhancing Multiculturality - TEEM'18, 788-793. New York, New York, USA: ACM Press. https://doi.org/10.1145/3284179.3284315

Fidalgo-Blanco, Ángel, Sein-Echaluce, M. L., \& GarcíaPeñalvo, F. J. (2017). APFT: Active peer-based Flip Teaching. ACM International Conference Proceeding Series, Part F1322. https://doi.org/10.1145/3144826.3145433

Fidalgo-Blanco, Ángel, Sein-Echaluce, M. L., \& GarcíaPeñalvo, F. J. (2018). Micro Flip Teaching with Collective Intelligence. In I. A. Zaphiris P. (Ed.), Learning and Collaboration Technologies. LCT 2018. Lecture Notes in Computer Science (pp. 400-415). Las Vegas: Springer, Cham. https://doi.org/https://doi.org/10.1007/978-3-319-91743$6 \_30$

García-Peñalvo, F. J., Fidalgo-Blanco, A., Sein-Echaluce, M. L., \& Sánchez-Canales, M. (2019). Active peer-based Flip Teaching: An active methodology based on RT$C I C L O$. IGI GLOBAL.

González Pérez, S., \& Fabiani Bendicho, M. de la P. (2019). Introduciendo la figura del estudiante-tutor en prácticas de ingeniería eléctrica y electrónica. In De los procesos de cambio al cambio con sentido (pp. 281-292). Servicio de publicaciones de la Universidad de la Laguna. https://doi.org/10.25145/b.innovaull.2019.021

Howard, J. L., Bureau, J., Guay, F., Chong, J. X. Y., \& Ryan, R. M. (2021). Student Motivation and Associated Outcomes: A Meta-Analysis From Self-Determination Theory. Perspectives on Psychological Science. https://doi.org/10.1177/1745691620966789

Jones, B. D. (2009). Motivating Students to Engage in Learning: The MUSIC Model of Academic Motivation. International Journal of Teaching and Learning in Higher Education, 21(2), 272-285. Retrieved from http://www.isetl.org/ijtlhe/ 
Jones, B. D. (2014). User Guide for Assessing the Components of the MUSIC Model of Academic Motivation. Retrieved September 2, 2021, from http://www.MotivatingStudents.info

Krathwohl, D. R. (2002). A Revision of Bloom's Taxonomy: An Overview. Theory into Practice, 41(5), 2012-2018.

Nonaka, I., \& Takeuchi, Hirotaka. (1995). The knowledgecreating company: how Japanese companies create the dynamics of innovation. Oxford University Press.

Sein-Echaluce, M. L., Fidalgo-Blanco, A., Esteban-Escaño, J., \& García-Peñalvo, F. (2017). The learning improvement of engineering students using peer-created complementary resources. International Journal of Engineering Education, 33(2). 